\title{
A Nonlinear Reactivity Method with Application to Accident-Tolerant Fuels
}

\author{
Jeremy A. Roberts, Max Langston, Daniel Nichols, Eric Schlaikjer, \\ Graham Schlaikjer, and Hitesh Bindra \\ Department of Mechanical and Nuclear Engineering, Kansas State University
}

\begin{abstract}
A nonlinear reactivity model with thermal-hydraulic feedback is presented for analysis of PWR fuel with various cladding materials. The simplified, batch-wise neutronic model requires batch reactivities and migration areas as predetermined functions of burnup, temperature (fuel and coolant), and any design parameters having neutronic significance (e.g., enrichment or cladding composition). Here, such functions are found by using symbolic regression, a unique approach that finds both a functional form and any model coefficients simultaneously. Core thermal-hydraulics are modeled using single-channel, axially-averaged values. The models were implemented in an opensource, Python-based tool, used here to the analyze fuel with Zr-based cladding having outer, protective layers of either $\mathrm{FeCrAl}$ or $\mathrm{SiC}$, two materials proposed for use in next-generation, accident-tolerant fuel.
\end{abstract}

\section{Keywords}

nonlinear reactivity model, nodal parameter model, coupled physics, accidenttolerant fuel, educational tools 


\section{INTRODUCTION}

Advanced cladding materials are of increasing interest for improved safety under a variety of planned and unplanned operating conditions. ${ }^{1}$ Although highresolution, coupled-physics models of these materials will be required before their adoption, a variety of parametric studies can be performed using simple, coupled-physics models to understand the gross impacts of a material on various reactor characteristics. This paper presents a coupled neutronics and thermalhydraulics tool ${ }^{2}$ based on a nonlinear reactivity model for analysis of PWR fuel and the results of its application to fuels with advanced $\mathrm{SiC}$ and FeCrAl cladding. While a full study would necessarily require inclusion of structural mechanics, the models presented here consider only neutronics and thermalhydraulics. With certain extensions, the models described can easily be extended to other applications. Similar studies have recently been conducted that focused primarily on the neutronics ${ }^{3}$ and structural mechanics ${ }^{4}$ of $\mathrm{FeCrAl}$ and/or $\mathrm{SiC}$ cladding. Although the results presented herein for illustration are consistent with those previously reported,,$^{3,4}$ the methods developed here provide a simple way to incorporate core-level thermal-hydraulics into the neutronic analysis.

The rest of this paper is organized as follows. Section II presents a nonlinear reactivity method that provides batch-wise neutronic detail based on precomputed parameters from lattice-physics calculations. These temperature-dependent parameters, described in Section III, provide direct coupling to a simple thermalhydraulics model, presented in Section IV. Section IV-C discusses the simple scheme used to couple the models, while Section V provides some illustrative results that demonstrate the potential utility of such simple models.

\section{Neutronic MOdeL}

A neutronic model that can capture core-level effects would be ideal for the analysis of cladding materials. Such models can provide assembly powers and, at 
least approximately, pin powers. Unfortunately, even the simplest of these models, e.g., the classical FLARE nodal method, ${ }^{5}$ require an explicit geometry and other detail that would preclude their use in parametric studies.

As an alternative, a simple model is proposed, which is based in part on the linear reactivity model (LRM). ${ }^{6}$ The basic premise of the model is that a core is comprised of one or more "batches" of like fuel, and the neutronic properties of these batches can be found by simple approximations based essentially on average values. The output of this model includes cycle-averaged batch powers and burnups, which can be coupled to a thermal-hydraulics model. LRM has long been used for various parametric studies, and an LRM-like method was recently used to study the impact of FeCrAl cladding. ${ }^{3}$

\section{A. Linear Reactivity Model}

The linear reactivity model is based on the assumptions that a core is composed of one or more batches of like fuel and that the reactivity of the fuel varies linearly with burnup, ${ }^{6}$ i.e.,

$$
\rho(B)=\rho_{0}-A B
$$

where $\rho$ is reactivity, $B$ is burnup (usually in $\mathrm{GWd} / \mathrm{MTU}$ ), and $\rho_{0}$ and $A$ are constants. Reactivity can also be defined as

$$
\rho=\frac{k-1}{k}
$$

where $k$ is the multiplication factor, i.e., the ratio of gains from fissions to losses by absorption and, if applicable, leakage.

In the simplest LRM approximation, the core reactivity is taken to be the arithmetic average of the various fuel reactivities. Suppose the core is composed of $N$ batches of equal size; for a typical PWR, $N \approx 3$. The core reactivity is then 
taken to be

$$
\bar{\rho}=\frac{1}{N} \sum_{i=1}^{N} \rho_{i}
$$

where $\rho_{i}$ is the reactivity of the $i$ th batch. Usually, the infinite multiplication factor $k_{\infty}$ is used, so $\rho_{i}$ represents the reactivity of an infinite reactor composed of the $i$ th-batch fuel. To include losses due to leakage, the core reactivity can be redefined as

$$
\bar{\rho}=\frac{1}{N} \sum_{i=1}^{N} \rho_{i}-\rho_{L}
$$

where $\rho_{L}$ is the reactivity penalty due to leakage and is typically close to $4 \%$.

The classical use of LRM is to estimate critical cycle lengths for various batching schemes. Consider an $N$-batch core at the end of cycle (EOC). The EOC reactivity of each batch is

$$
\begin{gathered}
\rho_{1}=\rho_{0}-A B_{c} \\
\rho_{2}=\rho_{0}-2 A B_{c} \\
\vdots \\
\rho_{N}=\rho_{0}-N A B_{c}
\end{gathered}
$$

where $B_{c}$ is the cycle length, i.e., the (average) burnup each batch accumulates between fuel reloads. The corresponding core average reactivity is

$$
\bar{\rho}=\frac{1}{N}\left(N \rho_{0}-\frac{N(N+1)}{2} A B_{c}\right)=\rho_{0}-\frac{N+1}{2} A B_{c} .
$$

If the EOC reactivity is assumed to be zero, then

$$
B_{c}=\frac{2 \rho_{0}}{(N+1) A}=\frac{2 B_{s}}{N+1},
$$

where $B_{s}=\rho_{0} / A$ is the single-batch cycle burnup. The batch burnup after $N$ 
cycles, often called the "discharge burnup," is

$$
B_{d}=N B_{c}=\frac{2 N B_{s}}{N+1}
$$

A key observation is that by using multiple fuel batches, one can extract more energy from the fuel than if the reactor were fueled with just one batch. In the extreme of continuous fuel reloading, $N \rightarrow \infty$, and the resulting discharge burnup is doubled.

\section{B. Nonlinear Reactivity Model}

Even though the LRM assumes that $\rho$ varies linearly with burnup, this assumption is unnecessary if numerical techniques are used to analyze fuel configurations. In other words, for $N$ batches, the cycle length $B_{c}$ can be found by solving the nonlinear equation

$$
\frac{1}{N} \sum_{i=1}^{N} \rho\left(i B_{c}\right)=\rho_{L}
$$

where leakage is included explicitly. Once $B_{c}$ is determined, batch-average properties can be evaluated for any burnup $B \in\left[0, B_{c}\right]$.

Of course, the reactivity $\rho_{i}$ of the $i$ th batch is a function not only of burnup but also of the cladding material (in the present study, a protective coating thickness), fuel and coolant temperatures, and boron concentration. Equation (9) (like Eq. (6)) is based on the implicit assumption that batch powers are equal. At any point during the cycle, the reactivity should be positive, and a corresponding critical boron concentration can be found by nonlinear iteration for any burnup of interest.

Nonlinear reactivity models (NRMs) have long been studied, usually with the assumption that $\rho$ still has a reasonably tractable dependence on $B$, e.g., a polynomial. ${ }^{6}$ Such a model based on equal-power sharing was used to accelerate scoping studies of fluoride salt-cooled reactors. ${ }^{7}$ In addition, a NRM based on a simple linear-power sharing model was used to study pebble-bed, gas-cooled 
reactors. ${ }^{8}$

1) Unequal Power Sharing: Although the model described above is adequate for some analyses, in reality the power of various batches should depend on the burnup. For instance, fresh fuel (if unpoisoned) should burn "hotter" than older, burned fuel, as it has more fissile material. In the following sections, a simple way to account for this observation is developed following the approach of Ref. 6.

Consider a core divided into $N$ regions (e.g., assemblies or batches). The reactivity of the $i$ th region is

$$
\rho_{i}=\frac{F_{i}-A_{i}}{F_{i}}
$$

where $F_{i}$ and $A_{i}$ are the region-wise neutron production and absorption rates, respectively. The reactivity of the whole core is

$$
\rho=\frac{\sum_{i=1}^{N}\left(F_{i}-A_{i}\right)}{\sum_{i=1}^{N} F_{i}}=\frac{\sum_{i=1}^{N} F_{i} \rho_{i}}{\sum_{i=1}^{N} F_{i}} .
$$

To a very good approximation, the region-wise neutron production rates $F_{i}$ can be replaced by the region-wise powers $q_{i}$, so that*

$$
\rho \approx \frac{\sum_{i=1}^{N} q_{i} \rho_{i}}{\sum_{i=1}^{N} q_{i}}=\frac{\sum_{i=1}^{N} q_{i} \rho_{i}}{Q},
$$

where $Q$ is the reactor power. Let

$$
f_{i}=\frac{q_{i}}{Q}
$$

denote the fraction of power generated in the $i$ th region, subject to

$$
\sum_{i=1}^{N} f_{i}=1
$$

\footnotetext{
${ }^{*}$ Note that $F=\nu \Sigma_{f} \phi$ while $q=\kappa \Sigma_{f} \phi$, where $\phi$ is the flux, $\Sigma_{f}$ is the fission cross section, $\nu$ is the average number of neutrons produced per fission, and $\kappa$ is the average energy released per fission. Over a wide range of burnups, the ratio $\kappa / \nu$ is relatively constant, which implies that $F \propto q$ is a good approximation.
} 
Note that $f_{i}$ is a function of burnup within a cycle because the relative assembly powers change with burnup. With substitution of Eq. (13) into Eq. (12), the core reactivity (neglecting leakage) is

$$
\rho=\sum_{i=1}^{N} f_{i} \rho_{i} .
$$

2) Determination of the Cycle Length: Let the EOC burnup of the $i$ th batch be denoted by $B_{i}$. This implies that $B_{N}=B_{d}$, i.e., the discharge burnup. Finally, let $B_{0}=0$. Then, the burnup accumulated by region $i$ during the cycle is given by

$$
B_{i}-B_{i-1}=\Delta B_{i}=B_{c} \times \frac{\bar{f}_{i}}{\bar{f}}
$$

where

$$
\begin{gathered}
\bar{f}_{i}=\frac{\int_{B_{i-1}}^{B_{i}} f_{i}(B) d B}{\Delta B_{i}}, \\
\bar{f}=\frac{1}{N}
\end{gathered}
$$

and the ratio $\bar{f}_{i} / \bar{f}$ is the cycle-averaged, region-wise, power peaking factor.

From Eqs. (16)-(18), the EOC reactivity of region $i$ is found to be

$$
\begin{aligned}
B_{i} & =B_{i-1}+\Delta B_{i} \\
& =B_{i-2}+\Delta B_{i-1}+\Delta B_{i} \\
& \vdots \\
& =B_{0}+\Delta B_{1}+\Delta B_{2}+\ldots+\Delta B_{i-1}+\Delta B_{i} \\
& =\sum_{j=1}^{i} \Delta B_{j} \\
& =N B_{c} \sum_{j=1}^{i} \bar{f}_{j} .
\end{aligned}
$$


Because $B_{N}=B_{d}=N B_{c}$, the region- $i$ burnup is

$$
B_{i}=B_{N} \sum_{j=1}^{i} \bar{f}_{j}
$$

and for $i=N$, this result indicates that

$$
\sum_{j=1}^{i} \bar{f}_{j}=1
$$

which is consistent with Eq. (14).

For a fixed leakage reactivity penalty $\rho_{L}$, the power-weighted, core-averaged, EOC reactivity must satisfy

$$
\bar{\rho}=\sum_{i=1}^{N} f\left(B_{i}\right) \rho\left(B_{i}\right)=\rho_{L} .
$$

Together, Eqs. (17), (20), and (22) define a nonlinear system of equations for $\mathbf{B}=\left[B_{1}, B_{2}, \ldots, B_{N}\right]$, i.e.,

$$
\mathbf{r}(\mathbf{B}) \equiv\left(\begin{array}{c}
B_{1}-B_{N} \bar{f}_{1} \\
B_{2}-B_{N}\left(\bar{f}_{1}+\bar{f}_{2}\right) \\
\ldots \\
B_{N-1}-B_{N}\left(\bar{f}_{1}+\bar{f}_{2}+\ldots+\bar{f}_{N-1}\right) \\
\sum_{i=1}^{N} f\left(B_{i}\right) \rho\left(B_{i}\right)-\rho_{L}
\end{array}\right)=\mathbf{0}
$$

which can be solved for the $B_{i}$ by an appropriate numerical scheme, e.g., Newton's method.

3) Estimation of Power Fractions: All that remains to complete the model is to define the region-wise power fractions $f_{i}$ in some way. The simplest approach is to assume values based on the results from a whole-core analysis. Such an approach was used in recent work on the assessment of $\mathrm{FeCrAl}$ cladding materials. ${ }^{3}$ Here, a way to estimate these power fractions directly from the neutronic properties of 
the batches is developed following the presentation of Ref. 6 .

Consider the two-group diffusion equations:

$$
\begin{aligned}
& -D_{1} \nabla^{2} \phi_{1}(\mathbf{r})+\Sigma_{r 1}(\mathbf{r}) \phi_{1}(\mathbf{r})=\frac{1}{k}\left[\nu \Sigma_{f 1}(\mathbf{r}) \phi_{1}(\mathbf{r})+\nu \Sigma_{f 2}(\mathbf{r}) \phi_{2}(\mathbf{r})\right] \\
& -D_{2} \nabla^{2} \phi_{2}(\mathbf{r})+\Sigma_{a 2}(\mathbf{r}) \phi_{2}(\mathbf{r})=\Sigma_{s 12}(\mathbf{r}) \phi_{1}(\mathbf{r})
\end{aligned}
$$

where upscatter is neglected, fission neutrons are limited to the fast group, and $\Sigma_{r 1}=\Sigma_{a 1}+\Sigma_{s 12}$ is the fast removal cross section. The remaining notation is standard. ${ }^{9}$

In the two-group approximation, the core reactivity is given by

$$
\rho=\frac{k-1}{k}=\frac{\left[\nu \Sigma_{f 1} \phi_{1}+\nu \Sigma_{f 2} \phi_{2}\right]-\left[\Sigma_{a 1} \phi_{1}+\Sigma_{a 2} \phi_{2}\right]}{\nu \Sigma_{f 1} \phi_{1}+\nu \Sigma_{f 2} \phi_{2}} .
$$

If thermal leakage is assumed to be negligible, i.e., $D_{2} \nabla^{2} \phi_{2} \ll \Sigma_{a 1} \phi_{2}$, then

$$
\phi_{2}=\frac{\Sigma_{12} \phi_{1}}{\Sigma_{a 2}}
$$

which is sometimes referred to as the "one-and-a-half group" approximation. ${ }^{9}$ By combining Eqs. (24), (26), and, (25), one has

$$
\nabla^{2} \phi_{1}+\frac{1}{M^{2}}\left(\frac{\rho}{1-\rho}\right) \phi_{1}=0,
$$

where the migration area $M^{2}$ is defined as

$$
M^{2}=\frac{D_{1}}{\Sigma_{s 12}+\Sigma_{a 1}} .
$$

The power density is related to the fast flux by

$$
q=\kappa\left(\Sigma_{f 1}+\frac{\Sigma_{f 2} \Sigma_{12}}{\Sigma_{a 2}}\right) \phi_{1}
$$

and, hence, Eq. (27) can be rewritten as

$$
\nabla^{2}[q(1-\rho)]+\frac{\rho q}{M^{2}}=0
$$


However, if this equation is discretized over assemblies (or sub-assemblies), the local $q_{i}$ for node $i$ can be cast instead as the power fraction $f_{i}$ times the total power $Q$. Because $Q$ is constant, it may be eliminated, leading to

$$
\nabla^{2}\left[f_{i}\left(1-\rho_{i}\right)\right]+\frac{\rho_{i} f_{i}}{M_{i}^{2}}=0
$$

Of course, the direct solution of this equation (via finite differences) would be about as difficult as solving the FLARE or similar nodal-diffusion equations. Moreover, such a model would require an explicit fuel map that requires input that would not likely exist in parametric scoping studies.

Here, a simpler approach is adopted. The Laplacian can be approximated based on the difference of the node $i$ value with neighboring values, but to simplify the analysis, the neighbors will be taken to have core-averaged values. Consider the 1-D case, with node $i$ surrounded by nodes $i-1$ and $i+1$. The Laplacian can be approximated by

$$
\begin{aligned}
& \nabla^{2}\left[f_{i}\left(1-\rho_{i}\right)\right] \\
& \quad \approx \frac{f_{i+1}\left(1-\rho_{i+1}\right)-2 f_{i}\left(1-\rho_{i}\right)+f_{i-1}\left(1-\rho_{i-1}\right)}{h^{2}},
\end{aligned}
$$

where $h$ is the width of the node. If the neighbor values, e.g., $f_{i+1}$ and $\rho_{i+1}$, are core-averaged values, then

$$
\frac{\bar{f}(1-\bar{\rho})-f_{i}\left(1-\rho_{i}\right)}{\frac{1}{2} h^{2}}+\frac{\rho_{i} f_{i}}{M_{i}^{2}}=0,
$$

Furthermore, if the core reactivity $\bar{\rho}$ is zero, i.e., the reactor is critical, then

$$
\frac{\bar{f}-f_{i}\left(1-\rho_{i}\right)}{\frac{1}{2} h^{2}}+\frac{\rho_{i} f_{i}}{M_{i}^{2}}=0 .
$$

and, hence,

$$
f_{i}=\frac{\bar{f}}{1-\rho_{i}\left(1+\frac{\frac{1}{2} h^{2}}{M_{i}^{2}}\right)}=\frac{\bar{f}}{1-\theta_{i} \rho_{i}}=\frac{1}{N\left(1-\theta_{i} \rho_{i}\right)}
$$


where

$$
\theta_{i}=1+\frac{\frac{1}{2} h^{2}}{M_{i}^{2}} .
$$

It is easy to show the factor of $1 / 2$ in $\theta_{i}$ changes to $1 / 4$ for $2-\mathrm{D}$ and $1 / 6$ for $3-\mathrm{D}$ grids. In all results presented in Section V, a 2-D grid was assumed. Although this model for $\theta$ comes directly from a reasonable physical model, in practice, it might be better to adjust $\theta$ to match results of more detailed models that include explicit spatial dependence.

Equation (35) is correct only when the core reactivity is zero, i.e., at EOC without a leakage penalty. In any other case, the resulting power fractions, which are functions of burnup, are not guaranteed to satisfy Eq. (14) and, hence, they must be normalized before use. This includes the computation of the region-wise, cycle-averaged values $\bar{f}_{i}$.

\section{Neutronic Data Model}

The nonlinear reactivity method presented in Section II depends on the availability of two nodal parameters, $\rho$ and $M^{2}$, which are assumed to be assemblyspecific functions of burnup, fuel temperature, coolant temperature, and boron concentration. Both of these (and other) parameters can be computed using standard lattice-physics tools.

Such nodal parameters are typically represented by functions of several historical variables (e.g., the average boron concentration up to an exposure point) and several instantaneous variables (e.g., the boron concentration at an exposure of interest, possibly different from past concentrations). For most productionlevel analyses, nodal parameters are tabulated for interpolation (e.g., PARCS ${ }^{10}$ or SIMULATE $^{11}$ ) or approximated by polynomial regression (e.g., NEXUS ${ }^{12}$ ).

For this work, the TRITON module of SCALE 6.1 was used to compute all nodal parameters. TRITON allows users to produce nodal parameters for baseline 
conditions as a function of burnup and for a variety of branch cases (i.e., step perturbations in state parameters such as the fuel temperature).

Let a generic nodal parameter be denoted by $p$ for a specific fuel assembly type. Then

$$
p \equiv f\left(B, T_{F}, T_{C}, C_{B}\right)
$$

where $B$ is the burnup (GWd/MTU), $T_{F}$ and $T_{C}$ are the current fuel and coolant temperatures $(\mathrm{K})$, and $C_{B}$ is the current boron concentration (ppm). Dependence on historical values is neglected. For the present work, the data is also dependent on the thickness of either SiC or FeAlCr, as described below.

To represent these dependencies on state parameters and cladding features, symbolic regression was employed using the Eureqa tool, ${ }^{13,14}$ which appears to be the first use of symbolic regression for the nodal parameter problem. Whereas traditional regression models seek to fit data to a particular functional form with undetermined coefficients, symbolic regression determines the form and the values of the coefficients. This process can be very time-consuming, but the results tend to represent data with fewer coefficients and operations, which, in turn, helps users understand their data better by isolating key dependencies. Although this approach might not be useful for production-level analyses, the types of functions found for various parameter types might be useful starting points for advanced (i.e., non-polynomial) regression.

Eureqa was used to provide fits of $\rho$ and $M^{2}$ to the data computed by TRITON. Two basic approaches were followed. The first applied Eureqa directly to fits having the form of Eq. (37). The second applied Eureqa to the approximate form

$$
\begin{aligned}
p & \equiv p_{o}(B)+\frac{\partial p}{\partial T_{F}}\left(T_{F}-T_{F o}\right) \\
& +\frac{\partial p}{\partial T_{C}}\left(T_{C}-T_{C o}\right)+\frac{\partial p}{\partial C_{B}}\left(C_{B}-C_{B o}\right),
\end{aligned}
$$

where $p_{o}(B)$ is a function of all non-state variables (e.g., burnup and cladding 
attributes) at baseline conditions, $\partial p / \partial x$ is the partial derivative of $p$ with respect to the state variable $x$ at nominal conditions, and $x_{o}$ is the baseline value of $x$. For both approaches, the objective was to minimize the average, absolute error between the model and data.

Experience with these two approaches suggests strongly that the latter approach based on partial derivatives leads to fits that are more accurate overall but suffer from increased complexity. Because Eureqa tends to produce fits with a limited number of terms, subtle dependencies were easily lost when pursuing the first "fit all-at-once" approach. Consequently, all results presented in Section V are based on the second approach.

\section{Thermal-Hydraulics Model}

The thermal-hydraulic model developed is a simple, single-channel model. ${ }^{15}$ Assembly-averaged powers are used to define an axial-averaged, bulk coolant temperature for each assembly. Then, by using a very simple fuel pin model, the average fuel temperature is computed for each assembly. Coupling of the thermal-hydraulic and neutronic models is performed by fixed-point iteration.

\section{A. Bulk Coolant Temperature}

If pressure and friction losses are neglected, energy balance along an axial channel with single-phase flow is approximately described by ${ }^{15}$

$$
\dot{m} c_{p} \frac{d T_{C}}{d z}=q^{\prime}(z)
$$

where $T_{C}$ is the bulk coolant temperature, $\dot{m}$ is the coolant mass-flow rate, $c_{p}$ is the specific heat capacity, which is assumed to be constant, and $q^{\prime}$ is the axial linear heat generation rate. Because the nonlinear reactivity method is based on an effectively two-dimensional representation, the $z$-dependence of $q^{\prime}$ must be 
assumed. Here, that dependence is taken to be

$$
q^{\prime}(z)=q_{o}^{\prime} \cos \left(\frac{\pi z}{H+2 \delta H}\right)
$$

where $q_{o}^{\prime}$ is the axial peak linear heat generation rate, $H$ is the active fuel height, and $\delta H$ is the extrapolation length at either end of the core. The axial average linear heat generation rate is defined as

$$
\begin{aligned}
\bar{q}^{\prime} & =H^{-1} \int_{-H / 2}^{H / 2} q^{\prime}(z) d z \\
& =\left(\frac{2 q_{o}^{\prime}(H+2 \delta)}{\pi H}\right) \sin \left(\frac{\pi H}{2 H+4 \delta}\right) .
\end{aligned}
$$

If the assembly power $q$ is known (from the neutronic model) then

$$
\begin{aligned}
q & =H \bar{q}^{\prime} \\
& =\left(\frac{2 q_{o}^{\prime}(H+2 \delta)}{\pi}\right) \sin \left(\frac{\pi}{2 H+4 \delta}\right),
\end{aligned}
$$

from which the axial peak linear heat generation rate is determined to be

$$
q_{o}^{\prime}=\frac{\pi q}{2(H+2 \delta)} \frac{1}{\sin \left(\frac{\pi}{2 H+4 \delta}\right)} .
$$

Substitution of Eq. 40 into Eq. 39 and integration of the result over the axial length leads to

$$
\begin{aligned}
& \dot{m} c_{p}\left(T_{C}(z)-T_{\text {in }}\right) \\
& =\int_{-H / 2}^{z} q_{o}^{\prime} \cos \left(\frac{\pi z^{\prime}}{H+2 \delta H}\right) \\
& =\left[\sin \left(\frac{\pi H}{2 H+4 \delta}\right)+\sin \left(\frac{\pi z}{H+2 \delta}\right)\right]\left(\frac{2 \delta+H}{\pi}\right) .
\end{aligned}
$$


or

$$
\begin{aligned}
T_{C}(z) & =T_{\text {in }}+\left(\frac{2 \delta+H}{\pi \dot{m} c_{p}}\right) \\
& \times\left[\sin \left(\frac{\pi H}{2 H+4 \delta}\right)+\sin \left(\frac{\pi z}{H+2 \delta}\right)\right]
\end{aligned}
$$

where $T_{\text {in }} \equiv T_{C}(-H / 2)$. It follows that the average coolant temperature (equal here to the mid-plane temperature) is defined as

$$
\begin{aligned}
\bar{T}_{C} & =T_{\text {in }}+\left(\frac{q_{o}^{\prime}(H+2 \delta)}{\pi \dot{m} c_{p}}\right) \sin \left(\frac{\pi H}{2 H+4 \delta}\right) \\
& =T_{\text {in }}+\frac{\bar{q}^{\prime} H}{2 \dot{m} c_{p}}
\end{aligned}
$$

where

$$
\bar{q}^{\prime}=\left(\frac{2 q_{o}^{\prime}(H+2 \delta)}{\pi H}\right) \sin \left(\frac{\pi H}{2 H+4 \delta}\right)
$$

\section{B. Fuel Temperature}

To compute the assembly-averaged fuel temperatures, a single, representative fuel pin model is used that assumes constant power and constant coefficients (e.g., thermal conductivity). The radial-averaged fuel pin temperature at a particular axial plane is defined as ${ }^{15}$

$$
T_{F}(z)=\frac{q_{\mathrm{pin}}^{\prime}(z)}{8 \pi k_{F}}+T_{F o}(z)
$$

where $q_{\text {pin }}^{\prime}=q^{\prime} / N_{p}, N_{p}$ is the number of pins in an assembly, $k_{F}$ is the fuel thermal conductivity, and $T_{F o}$ is the temperature at the outer surface of the fuel. The total temperature drop across the gas gap, cladding, and between the outer cladding surface and the bulk coolant is ${ }^{15}$

$$
\begin{aligned}
\Delta T(z) & =\frac{q_{\text {pin }}^{\prime}(z)}{2 \pi}\left(\frac{t_{g}}{r_{g} k_{g}}+\frac{\ln \left(r_{c o} / r_{c i}\right)}{k_{c}}+\frac{1}{r_{c o} h}\right) \\
& \equiv \frac{q_{\text {pin }}^{\prime}(z)}{k_{N F}},
\end{aligned}
$$


where $t_{g}$ is the gap thickness, $r_{g}$ is the average gap radius, $r_{c i}$ and $r_{c o}$ are the inner and outer cladding radii, $k_{g}$ and $k_{c}$ are the gap and cladding thermal conductivities, $h$ is the coolant heat transfer coefficient, and $k_{N F}$ is the effective thermal conductivity for all non-fuel components. Given the bulk moderator temperature $T_{C}(z)$, the radial-averaged fuel temperature reduces to

$$
T_{F}(z)=\frac{q_{\text {pin }}^{\prime}(z)}{8 \pi k_{F}}+T_{C}(z)+\Delta T(z),
$$

the axial average of which is defined as

$$
\bar{T}_{F}=\bar{T}_{C}+\bar{q}_{\text {pin }}^{\prime}\left(\frac{1}{4 \pi k_{F}}+\frac{1}{k_{N F}}\right) .
$$

Thermal conductivities are generally functions of temperature and, in the case of fuel, burnup. Here, only the axially- and radially-averaged temperatures are used to evaluate conductivities. A straightforward extension to the model would admit radial and axial dependence of the temperature and, hence, conductivities.

\section{Coupling Neutronics and Thermal-Hydraulics}

Coupling of the nonlinear reactivity and thermal-hydraulics models is straightforward by using a fixed-point iteration. To perform the iteration, various parameters must be defined a priori, including the average assembly thermal power. Because each batch is normalized to single-assembly values, the average batch power is taken to be the average assembly power.

Initial values for the bulk coolant and fuel temperatures for each batch are assumed to be known. With these temperatures, the neutronic properties are evaluated, and the batch-wise cycle burnups and power-peaking factors are computed. The power-peaking factors are in turn provided to the thermal-hydraulics model. Based on these fixed batch-wise powers, new temperatures are computed, and the process repeats until temperatures are converged to within a user-defined tolerance. 
For the problems studied, convergence to within $0.01 \mathrm{~K}$ was achieved in fewer than 10 iterations.

\section{NUMERICAL DEMONSTRATION}

Zirconium-based alloys (e.g., Zr-4) are currently used for PWR fuel cladding, but owing to their behavior under accident conditions, alternative, accident-tolerant cladding materials are of interest. Here, two such materials were considered: Fe$\mathrm{CrAl}(21 \% \mathrm{Cr}, 5.8 \% \mathrm{Al}, 0.7 \% \mathrm{Si}, 0.4 \% \mathrm{Mn}$, and $0.08 \% \mathrm{C}$ by mass $)$ and SiC. Both $\mathrm{FeCrAl}$ and $\mathrm{SiC}$ may offer enhanced damage resistance under accident conditions. However, each cladding has potential disadvantages. For instance, $\mathrm{FeCrAl}$ is less neutron transparent than $\mathrm{Zr}$ cladding. This means that more neutrons are absorbed in the $\mathrm{FeCrAl}$, thus requiring increased enrichment to compensate for these losses. On the other hand, $\mathrm{SiC}$ is more neutron-transparent than $\mathrm{Zr}$, but it is a ceramic and, thus, is more brittle and prone to cracking. ${ }^{16}$ As an alternative to singlematerial, monolithic cladding based on $\mathrm{FeCrAl}$ or $\mathrm{SiC}$ alone, it has been proposed to use a thin layer of either material on a $\mathrm{Zr}$-based substrate. Here, thicknesses of 100 and 300 microns of either material on top of a $\mathrm{Zr}-4$ substrate were considered. In addition, monolithic (570 micron) cladding of each of the three materials was considered. An assessment of the structural mechanics implications of such layering schemes is beyond the present scope but has been examined elsewhere. ${ }^{16}$ All material properties for the cladding materials are consistent with those previously reported. ${ }^{16}$

Several numerical examples are presented below using the Python-based, opensource NRM tool available online. ${ }^{2}$ All files to produce the figures in this paper are included in the source code. 
TABLE I. ASSEMBLY AND FUEL-PIN DIMENSIONS

\begin{tabular}{ll}
\hline Assembly pitch & $21.5036 \mathrm{~cm}$ \\
Pin pitch & $1.2598 \mathrm{~cm}$ \\
Fuel pellet OR & $0.4096 \mathrm{~cm}$ \\
Clad IR & $0.4180 \mathrm{~cm}$ \\
Clad OR & $0.4750 \mathrm{~cm}$ \\
Guide/instrument tube IR & $0.5610 \mathrm{~cm}$ \\
Guide/instrument tube OR & $0.6120 \mathrm{~cm}$ \\
\hline
\end{tabular}

\section{Generation of Model Data}

The geometries considered are all based on a $17 \times 17$ Westinghouse-like design with dimensions and other characteristics drawn from the publicly-available literature and summarized in a publicly-available EPRI report. ${ }^{17}$ Fuel-pin dimensions are specified in Table I. All models were set to $4 \%$ initial enrichment with

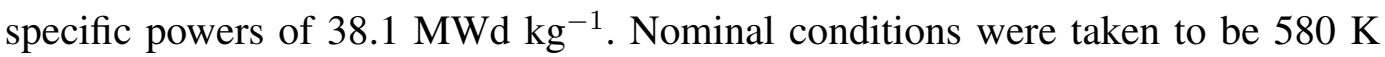
( $\rho=0.712 \mathrm{~g} \mathrm{~cm}^{-3}$ ) for the coolant temperature, $900 \mathrm{~K}$ for the fuel temperature, and $900 \mathrm{ppm}$ for the boron concentration. Branch cases consisted of coolant temperatures of $560 \mathrm{~K}\left(\rho=0.752 \mathrm{~g} \mathrm{~cm}^{-3}\right)$ and $600 \mathrm{~K}\left(\rho=0.660 \mathrm{~g} \mathrm{~cm}^{-3}\right)$, fuel temperatures of $800 \mathrm{~K}$ and $1000 \mathrm{~K}$, and boron concentrations of $0 \mathrm{ppm}$ and 1800 ppm.

The cladding temperature was taken to be

$$
T_{\text {clad }}=0.12 T_{F}+0.88 T_{C},
$$

where $T_{F}$ and $T_{C}$ are the fuel and coolant temperatures, respectively. ${ }^{17}$ The gas gap was filled with dry air at a density of $0.001 \mathrm{~g} \mathrm{~cm}^{-3}$ and temperature of $580 \mathrm{~K}$.

To minimize computational time, the TRITON models were simplified by depleting all fuel pins as lumped fuel. To quantify the error introduced by this approximation, a separate case was studied for which each fuel pin was depleted 
individually. The error in $k_{\infty}$ was less than $0.01 \%$ over a small range of burnups, which suggests the approximation is sufficiently accurate for the present study.

All of the fuel and cladding thermal conductivities used are consistent with those reported elsewhere ${ }^{16}$ except that the NFIR model ${ }^{18}$ for $\mathrm{UO}_{2}$ conductivity was used. A major limitation of the model is its treatment of the gas gap, which uses the following approximation for the gap conductance $h\left(\mathrm{~W} \mathrm{~m}^{-2} \mathrm{~K}^{-1}\right)$ based on Figure 8.24 of Ref. 15:

$$
h= \begin{cases}6000+166.7 / B & B<30 \\ 11000 & \text { otherwise }\end{cases}
$$

A better approach would use a fuel performance code (e.g., BISON) to generate correlations for the gap conductance as functions of applicable state variables. Such correlations would be consistent with the approach taken for neutronics modeling.

\section{Example 1: Effect of Cladding on Reactivity}

As a first example, the effect of the cladding material on the reactivity was assessed. Figure 1 shows the infinite multiplication factor as a function of burnup for five different cladding configurations: pure $\mathrm{Zr}-4$, two different $\mathrm{FeCrAl}$ thicknesses, and two different $\mathrm{SiC}$ thicknesses. The sharp drop in $k_{\infty}$ at very low burnups results from inclusion of equilibrium xenon (at nominal specific power).

Evidently, by replacing a portion of $\mathrm{Zr}-4$ with a Fe-based alloy, the reactivity is significantly reduced, as expected. Contrarily, an addition of $\mathrm{SiC}$ leads to a slightly increased reactivity. The relative impacts of $\mathrm{FeCrAl}$ and $\mathrm{SiC}$ are easily explained by noting the relative increase in $\Sigma_{a}$ caused by replacing $\mathrm{Zr}-4$ with FeCrAl is larger than the corresponding decrease caused by replacing $\mathrm{Zr}-4$ with SiC. Figure 2 shows the reactivity difference due to 100 and 300 micron layers of either FeCrAl or $\mathrm{SiC}$ relative to $\mathrm{Zr}-4$. The $\mathrm{FeCrAl}$ penalty ranges from approximately $-1000 \mathrm{pcm}$ 


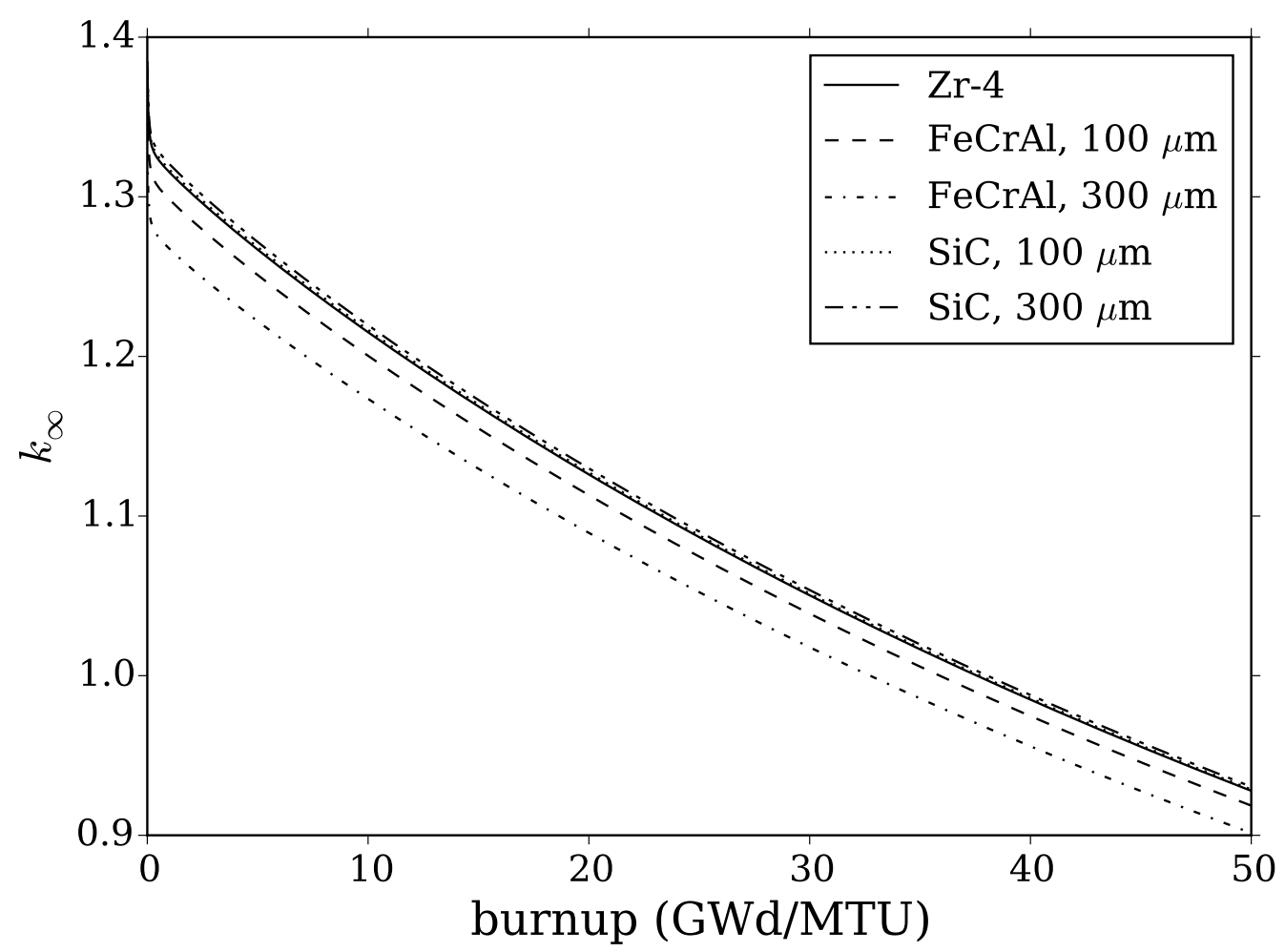

Fig. 1. Comparison of $k_{\infty}$ vs burnup for various cladding configurations.

to $-4000 \mathrm{pcm}$, whereas the gain from $\mathrm{SiC}$ is much smaller by nearly an order of magnitude. Defects for either cladding material are reduced in magnitude over the cycle.

\section{Example 2: Effect of Cladding on Cycle Length}

Because FeCrAl reduces the reactivity, its use can be expected to reduce the cycle length, perhaps compensated only by an increased enrichment. Similarly, use of $\mathrm{SiC}$ might actually lead to an increased cycle length (based on neutronics alone). To quantify these impacts, the cycle length was computed as a function of $\mathrm{FeCrAl}$ and $\mathrm{SiC}$ for a three-batch core assuming both equal-weighted and 


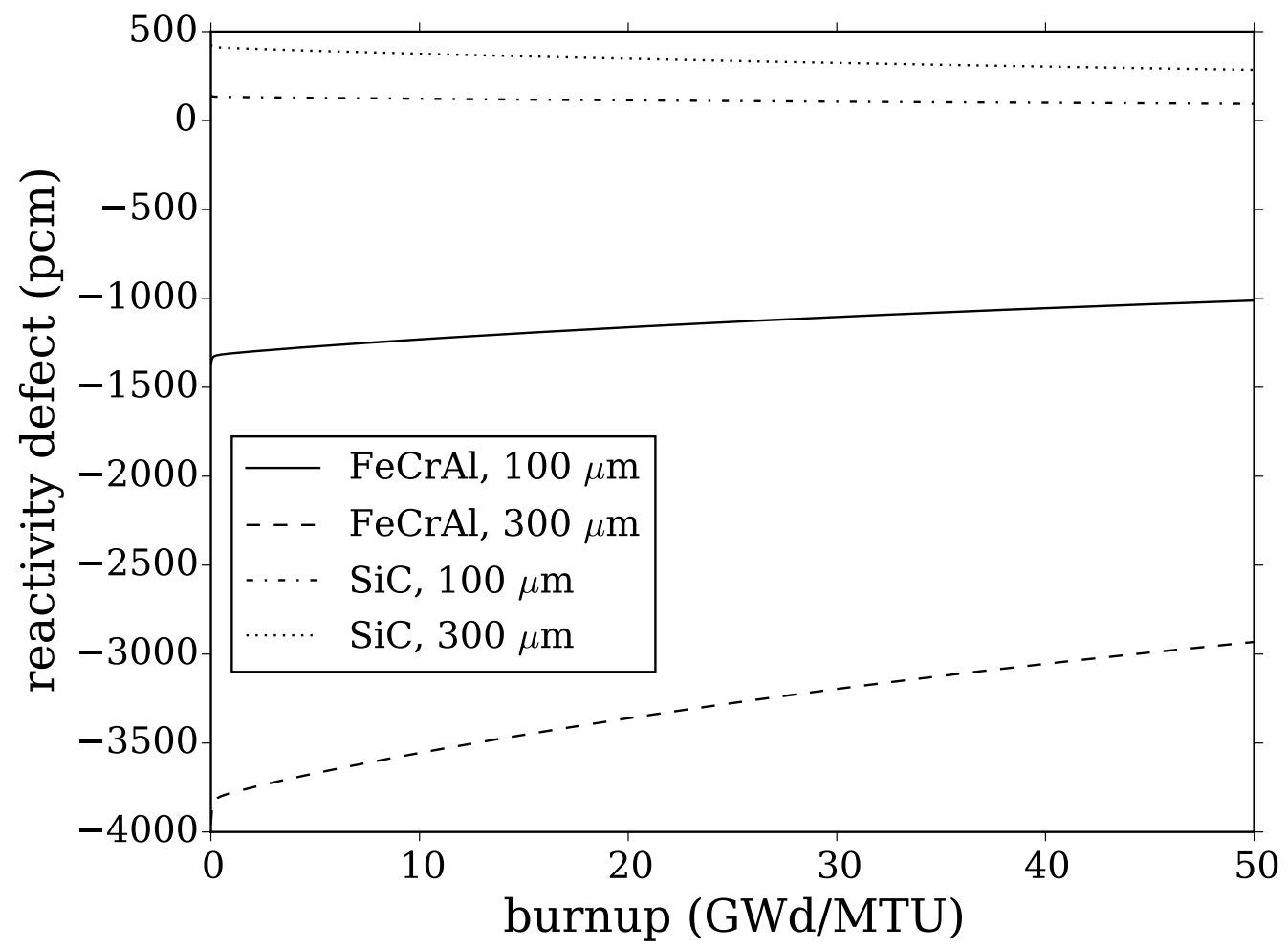

Fig. 2. Reactivity defect due to layer of $\mathrm{FeCrAl}$ or SiC.

reactivity-weighted power-sharing. A boron concentration of zero and a leakage penalty $\rho_{L}=0.04$ was assumed. The results are shown in Figure 3 .

Two observations can be made. First, the FeCrAl thickness has a much stronger effect on the cycle length than the $\mathrm{SiC}$ thickness, consistent with its larger impact on reactivity. Second, there is a marked difference in cycle lengths between the two power-sharing methods. Although parameters used in the reactivity-weighted approach are subject to tuning, the apparent sensitivity of the cycle length to the power-sharing method suggests that a realistic power profile (i.e., not uniform) may be important for capturing gross core behavior. 


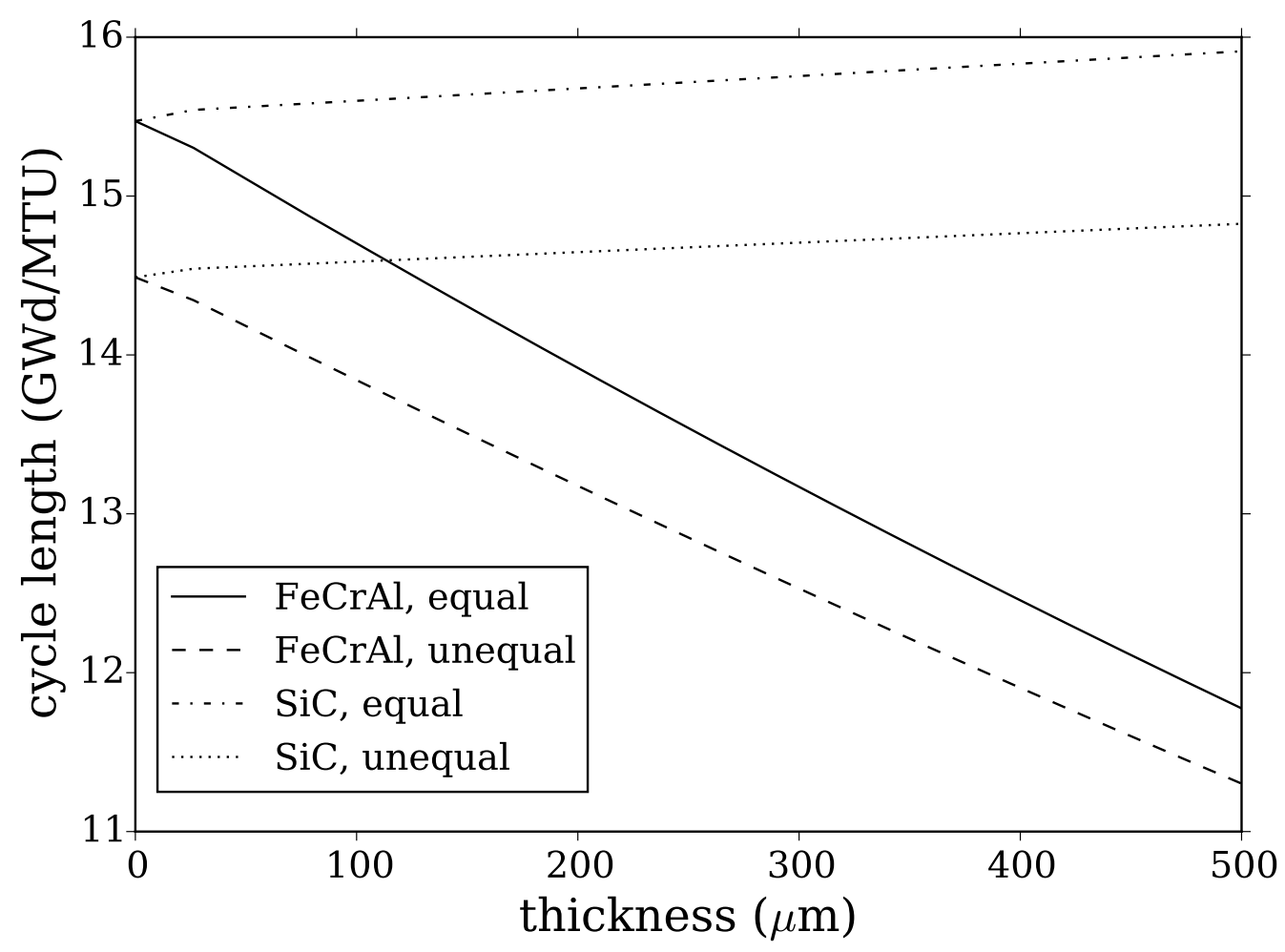

Fig. 3. Comparison of cycle lengths for various $\mathrm{FeCrAl}$ and $\mathrm{SiC}$ thicknesses.

\section{Example 3: Effect of Cladding on Batch Temperatures}

As a final example, the effect of cladding material on the batch-wise, cycleaveraged temperatures was studied. Reactivity-based power sharing leads to different batch-wise powers, and hence, temperatures. The batch-wise fuel temperatures for the same conditions as used in Example 2 were computed as a function of layer thickness. Results are shown in Figure 4. The corresponding coolant temperatures and power-peaking factors are shown in Figures 5 and 6, respectively.

From Figure 4, it is apparent that the FeCrAl cladding leads to a reduction in the average fuel temperatures. This effect is easily explained by the neutronic characteristics of FeCrAl: it reduces the batch reactivity and, hence, the batch 


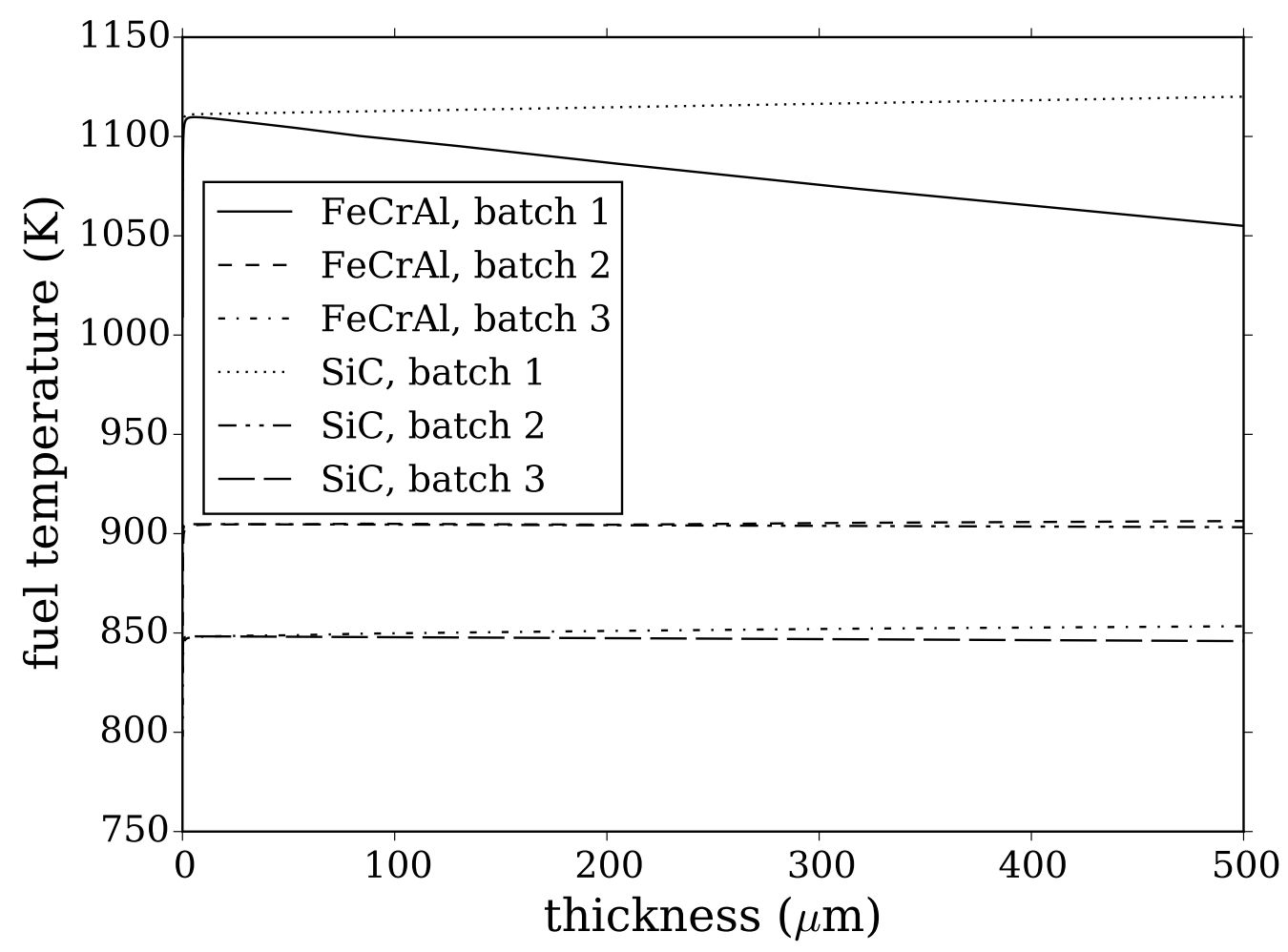

Fig. 4. Comparison of batch-wise, cycle-averaged fuel temperatures for various $\mathrm{FeCrAl}$ and SiC thicknesses.

power is also reduced. A very slight trend in the opposite direction is observed for thick SiC layers. The bulk coolant temperatures shown in Figure 5 generally follow the fuel-temperature trends, as do the power-peaking factors shown in Figure 6.

\section{CONCLUSION}

The models presented are admittedly very simple. However, once the underlying neutronic and thermal-hydraulic parameters are defined for the fuel configurations of interest, parametric studies like those of Section V are straightforward to conduct. An obvious path forward is the inclusion of a fuel-performance model. Although the models presented could, in theory, be coupled to detailed models 


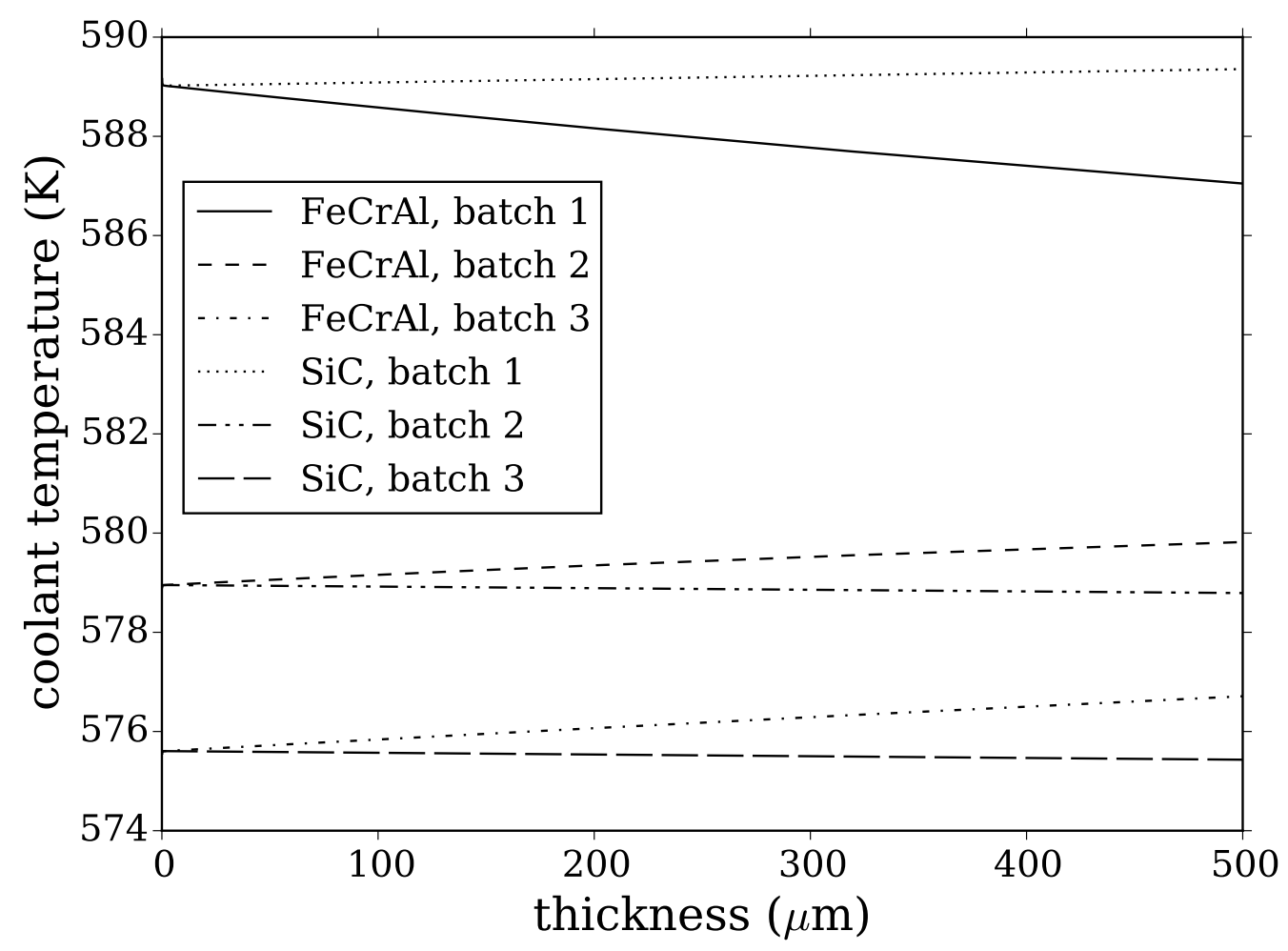

Fig. 5. Comparison of batch-wise, cycle-averaged coolant temperatures for various $\mathrm{FeCrAl}$ and $\mathrm{SiC}$ thicknesses.

like BISON, it would seem more natural to use a detailed model to generate data for a simplified model, much like what has been done here for the neutronic model. Of course, further improvements in the neutronic and thermal-hydraulic models are also possible, including tuning the neutronic model to higher-fidelity results and numerically computing the temperatures, e.g., to capture gradients in the thermal conductivities.

\section{ACKNOWLEDGMENTS}

The material presented is based upon work supported in part by the U.S. Department of Energy via Idaho National Laboratory under Prime Contract No. 


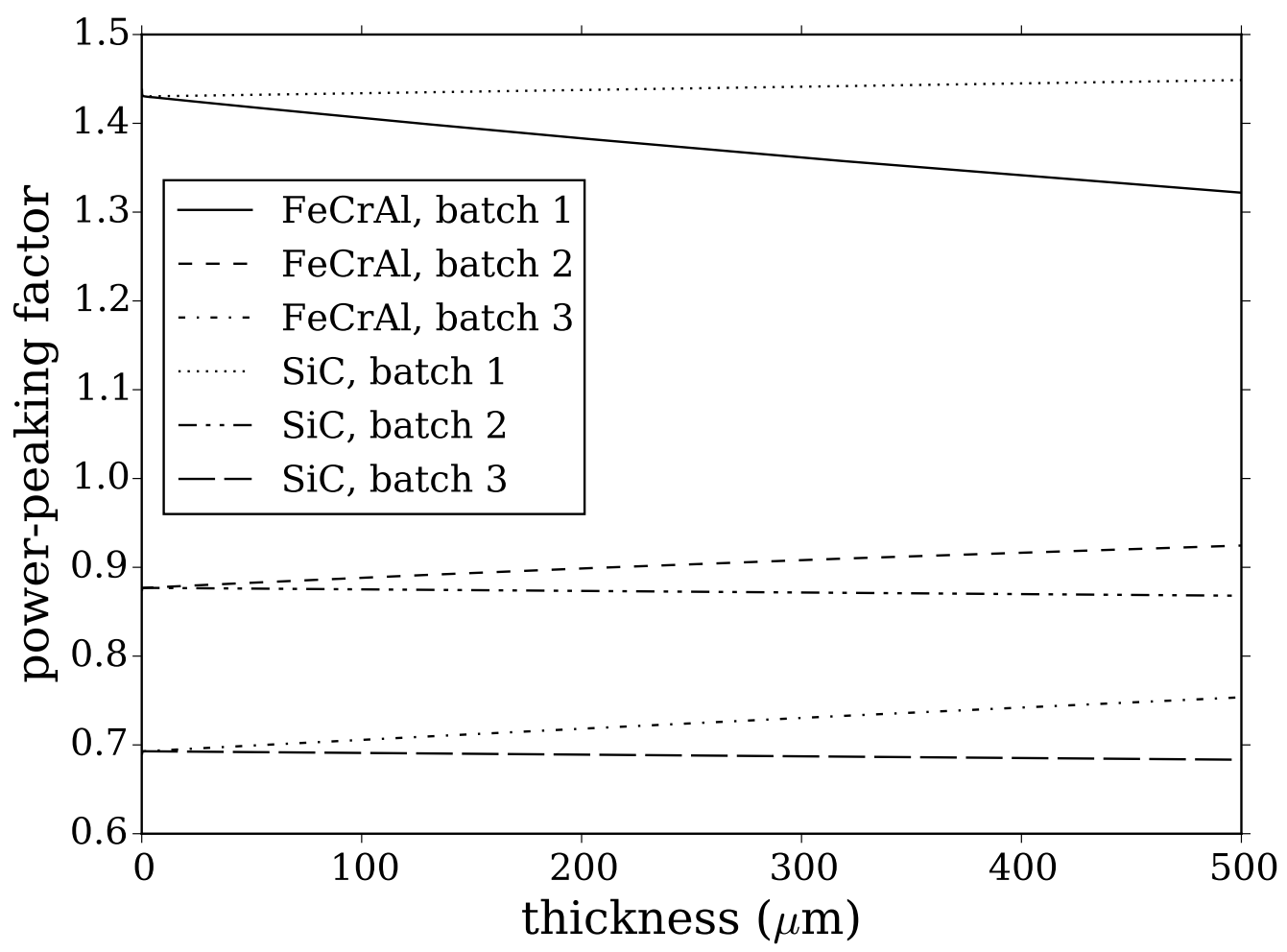

Fig. 6. Comparison of batch-wise, cycle-averaged power-peaking factors for various $\mathrm{FeCrAl}$ and $\mathrm{SiC}$ thicknesses.

DE-AC07-05ID14517. In addition, the work of M.L., D.N., E.S., and E.G. was further supported by KSU's Department of Mechanical and Nuclear Engineering Undergraduate Research Program.

\section{REFERENCES}

[1] S.M. Bragg-Sitton and W.J. Carmack. Update on the DOE's ATF Development Program. Nuclear News, December 2015.

[2] J. Roberts. NRM: A Coupled Neutronics and Thermal-Hydraulics Model Based on a Nonlinear Reactivity Model. corps-g.github.io/nrm, 2015.

[3] N.M. George, K. Terrani, J. Powers, A. Worrall, and I. Maldonado. Neutronic Analysis of Candidate Accident-Tolerant Cladding Concepts in Pressurized Water Reactors. Annals of Nuclear Energy, 75, 2015. 
[4] X. Wu, T. Kozlowski, and J.D. Hales. Neutronics and Fuel Performance Evaluation of Accident Tolerant FeCrAl Cladding Under Normal Operation Conditions. Annals of Nuclear Energy, 2015.

[5] D.L. Delp, J.M. Harriman, and M.J. Stedwell. FLARE-A Three-Dimensional Boiling Water Reactor Simulator. Technical report, General Electric Co. Atomic Power Equipment Dept., San Jose, Calif., 1964.

[6] M.J. Driscoll, T.J. Downar, and E.E. Pilat. The Linear Reactivity Model for Nuclear Fuel Management. American Nuclear Society, 1990.

[7] A.T. Cisneros and D. Ilas. Neutronics and Depletion Methods for Parametric Studies of Fluoride Salt Cooled High Temperature Reactors with Slab Fuel Geometry and Multi-Batch Fuel Management Schemes. Nuclear Technology, 183(3), 2013.

[8] Y. Tombakoğlu M. Yüksel, Z. Çeçen. Fuel Cycle Optimization using the Nonlinear Reactivity Model. In Proceedings of the International Conference Nuclear Energy for New Europe, Kranjska Gora, Slovenia, September 2002.

[9] W.M. Stacey. Nuclear Reactor Physics. John Wiley \& Sons, 2007.

[10] Y. Xu and T. Downar. Code for Generating the PARCS Cross Section Interface File PMAXS. Technical report, 2006.

[11] D. Knott and A. Yamamoto. Lattice Physics Computations. In Handbook of Nuclear Engineering, pages 913-1239. Springer, 2010.

[12] E. Müller, L. Mayhue, and B. Zhang. Reactor Physics Methods Development at Westinghouse. In Proceedings of the International Conference Nuclear Energy for New Europe, Portorov, Slovenia, September 2007.

[13] M. Schmidt and H. Lipson. Distilling Free-form Natural Laws from Experimental Data. Science, 324(5923):81-85, 2009.

[14] M. Schmidt and H. Lipson. Eureqa (version 0.98 beta). www.nutonian.com, 2014.

[15] N.E. Todreas and M.S. Kazimi. Nuclear Systems Volume 1: Thermal Hydraulic Fundamentals. Taylor \& Francis, second edition, 2011.

[16] X. Wu, P. Sabharwall, J. Hales, and T. Kozlowski. Neutronics and Fuel Performance Evaluation of Accident Tolerant Fuel Under Normal Operation Conditions. Technical Report INL/EXT-14-32591, Idaho National Laboratory, 2014.

[17] A. Machiels. Benchmarks for Quantifying Fuel Reactivity Depletion Uncertainty. EPRI Electric Power Research Institute, August 2011.

[18] J.D. Hales, S.R. Novascone, G. Pastore, D.M. Perez, B.W. Spencer, and R.L. Williamson. BISON Theory Manual: The Equations Behind Nuclear Fuel Analysis. Technical Report INL/EXT-13-29930, Idaho National Laboratory, 2013.

January 11, 2016

DRAFT 